\title{
Leukemia inhibitory factor is involved in the pathogenesis of NSCLC through activation of the STAT3 signaling pathway
}

\author{
HUAYING WANG ${ }^{1}$, SAI'NV SI $^{1}$, MINGJUN JIANG $^{2}$, LIPING CHEN $^{1}$, KEFENG HUANG $^{2}$ and WANJUN YU ${ }^{1}$ \\ Departments of ${ }^{1}$ Respiratory and Critical Care Medicine, and ${ }^{2}$ Thoracic Surgery, People's Hospital \\ Affiliated to Ningbo University, Yinzhou People's Hospital, Ningbo, Zhejiang 315040, P.R. China
}

Received July 23, 2020; Accepted May 4, 2021

DOI: $10.3892 / 01.2021 .12924$

\begin{abstract}
Leukemia inhibitory factor (LIF) is a tumor promoter in several cancer types. However, the role of LIF in non-small cell lung cancer (NSCLC) remains to be explored. The present study explored the hypothesis that LIF is important for NSCLC development by measuring LIF expression and its downstream signal transducer and activator of transcription 3 (STAT3) phosphorylation in tumor samples derived from patients with NSCLC. The association between LIF expression and clinical features was analyzed in two cancer subtypes. The effects of LIF on cell proliferation, migration and invasion were also evaluated in a NSCLC-derived cell line, A549. LIF mRNA and protein expression levels were significantly higher in tumor tissues compared with those in the corresponding adjacent and normal lung tissues. Regarding NSCLC subtypes, LIF expression was significantly higher in adenocarcinoma than in squamous cell carcinoma tissues. It was also found that phosphorylated-STAT3 levels were higher in tumor tissues compared with those in the corresponding adjacent and normal lung tissues, which was in agreement with the LIF expression levels in NSCLC tissues. Clinically, overexpression of LIF was positively correlated with aggressive tumor characteristics, including lymph node metastasis and advanced tumor stage. In A549 cells, LIF treatment enhanced cell proliferation, migration and invasion.
\end{abstract}

Correspondence to: Dr Wanjun Yu, Department of Respiratory and Critical Care Medicine, People's Hospital Affiliated to Ningbo University, Yinzhou People's Hospital, 251 East Baizhang Road, Ningbo, Zhejiang 315040, P.R. China

E-mail: nbyuwanjun@163.com

Abbreviations: LIF, leukemia inhibitory factor; NSCLC, non-small cell lung cancer; STAT3, signal transducer and activator of transcription 3; JAK, Janus tyrosine kinase; ERK, extracellular signal-regulated protein kinase; PI3K, phosphoinositide 3-kinase; IHC, immunohistochemistry; DMEM, Dulbecco's modified Eagle's medium; FBS, fetal bovine serum; CCK-8, Cell Counting Kit-8

Key words: leukemia inhibitory factor, non-small cell lung cancer, adenocarcinoma, squamous cell carcinoma, STAT3, migration, invasion
LIF also increased STAT3 phosphorylation in A549 cells, and the STAT3 inhibitor Stattic decreased A549 cell migration and invasion following LIF stimulation. The present results demonstrate that LIF is overexpressed in NSCLC, and that LIF can promote NSCLC development through activation of the STAT3 signaling pathway. The present study indicates that LIF may serve as a potential prognostic marker for NSCLC.

\section{Introduction}

Lung cancer is a leading cause of cancer-associated mortality. There are $>2$ million newly diagnosed lung cancer cases and 1.8 million mortalities from lung cancer each year worldwide (1). Non-small cell lung cancer (NSCLC) accounts for $85 \%$ of all lung cancer cases (2). Lung cancer treatments include surgery, radiotherapy, chemotherapy, targeted therapies and immunotherapies (3). However, despite the technological advances in recent years, the 5-year survival rate of patients with NSCLC remains low (14\%) (4). Poor responsiveness to therapies for NSCLC is associated with complex underlying mechanisms (5). Thus, understanding the molecular mechanism of NSCLC invasion and metastasis is important for identifying novel therapeutic targets and prognostic biomarkers to improve treatments of patients with NSCLC.

Leukemia inhibitory factor (LIF) is a multifunctional cytokine that belongs to the interleukin- 6 family of cytokines; it is expressed in numerous types of tissues and cells, such as embryonic stem cells and monocytes (6). Binding of LIF to its receptor activates critical signaling pathways that regulate cell proliferation, survival and differentiation. These pathways include the Janus tyrosine kinase (JAK)/signal transducer and activator of transcription 3 (STAT3), extracellular signal-regulated protein kinase (ERK) and phosphoinositide 3-kinase (PI3K) signaling pathways (7-9). LIF can play opposing roles in different types of cancer. For example, LIF can promote tumor growth in rhabdomyosarcoma (10), nasopharyngeal carcinoma (11), colorectal cancer (12) and oral squamous cell carcinoma (13), whereas it acts as a tumor suppressor in breast cancer $(14,15)$, melanoma (16) and hepatocellular carcinoma (17). However, the expression and role of LIF in NSCLC are largely unknown; thus, further studies are needed.

The present study determined the expression of LIF and the activation of its downstream signaling molecule STAT3 in tissues derived from patients with NSCLC. The association 
between LIF expression and the clinicopathological features of patients with NSCLC was analyzed to evaluate the role of LIF in NSCLC development, the effects of LIF treatment on cell proliferation, migration and invasiveness were measured in a NSCLC-derived cell line.

\section{Materials and methods}

Tissue specimen collection. Paraffin-embedded tissues from 105 patients with adenocarcinoma or squamous cell carcinoma, as confirmed by pathological biopsy at People's Hospital Affiliated to Ningbo University (Ningbo, China) were collected in the present study and underwent immunohistochemical analysis. Patient ages ranged from 38 to 80 years old (mean age, 60.79 years). Informed consent was provided by all patients based on the established protocol approved by the Ethics Committee of Yinzhou People's Hospital.

Specimens of tumor, adjacent and normal tissues from 33 patients with NSCLC were collected from January 2018 to January 2019 at Yinzhou People's Hospital. Tissue $2 \mathrm{~cm}$ away from the tumor was considered adjacent, while tissue $>5 \mathrm{~cm}$ away from the tumor was considered normal tissue. The specimens were flash frozen in liquid nitrogen immediately after surgical resection and stored at $-80^{\circ} \mathrm{C}$. All specimens were pathologically confirmed as one of two subtypes of NSCLC: Adenocarcinoma or squamous cell carcinoma. Frozen specimens were used to detect LIF mRNA and protein expression levels, as well as the expression levels of phosphorylated (p)-STAT3.

Immunohistochemistry (IHC). IHC for LIF and p-STAT3 was performed as previously described (18). Briefly, paraffin-embedded tissue sections were baked at $62^{\circ} \mathrm{C}$ for 30 min, deparaffinized in xylene (Sinopharm Chemical Reagent Co., Ltd.) and rehydrated in ethanol prior to pretreatment with $3 \%$ hydrogen peroxide/methanol solution (Sinopharm Chemical Reagent Co., Ltd.) for 15 min to block endogenous peroxidase activity. After blocking with $10 \%$ normal goat serum (cat. no. ST023; Beyotime Institute of Biotechnology, Inc.) for $30 \mathrm{~min}$ at room temperature, the tissue sections were incubated with anti-LIF (1:400 dilution; NBP2-27406; Novus Biologicals, Ltd.) and anti-p-STAT3 (Tyr705; 1:400 dilution; CST9145; Cell Signaling Technology, Inc.) primary antibodies overnight at $4^{\circ} \mathrm{C}$. The tissue sections were then treated with a biotinylated goat anti-rabbit (1:400 dilution; cat. no. BA1000; Vector Laboratories, Inc.) or goat anti-rat (1:400 dilution; cat. no. BA9400; Vector Laboratories, Inc.) secondary antibodies at $37^{\circ} \mathrm{C}$ for $30 \mathrm{~min}$. Immunoreactivity was visualized with a VECTASTAIN Elite ABC kit (Vector Laboratories, Inc.). The results of IHC were analyzed by two pathologists independently.

For evaluation of LIF expression, two pathologists randomly selected five areas of each slice under a microscope (Axio Lab.A1; Zeiss AG) with a 10X objective according to the distribution and intensity of LIF staining ( 0 for no expression, 1 for weak expression, 2 for moderate expression and 3 for high expression). The mean value of the five areas was used as the final score for each slice.

The expression of p-STAT3 was analyzed using ImageJ software (v.1.8.0.112; National Institutes of Health). Five areas of each slice were selected under a microscope (Axio Lab A1; Zeiss AG) with a $10 \mathrm{X}$ objective, and cells with positive staining in the nuclei were counted. The score was determined according to the percentage of positive cells ( 0 for no staining, 1 for $<10 \%$ staining, 2 for $11-50 \%$ staining and 3 for $>50 \%$ staining) and averaged. A mean value of $0-0.4$ was considered as non-expression, 0.5-1.4 as weak expression, 1.5-2.4 as moderate expression group and 2.5-3 as high expression $(13,19)$.

Western blot analysis. A standard western blot technique was used to detect protein expression in the tumor, adjacent and normal lung tissues, as well as in A549 cells. The tissues and A549 cells were collected in ice-cold RIPA lysis buffer (cat. no. P0013B; Beyotime Institute of Biotechnology, Inc.) containing protease inhibitor and phosphatase inhibitors (cat. no. A32961; Thermo Fisher Scientific, Inc.), and then centrifuged at $13,000 \times \mathrm{g}$ for $30 \mathrm{~min}$ at $4^{\circ} \mathrm{C}$. The concentration of homogenate protein was determined using a BCA protein assay kit (cat. no. P0012; Beyotime Institute of Biotechnology, Inc.). Samples $(30 \mu \mathrm{g})$ were loaded on $10 \%$ gels for SDS-PAGE and separated proteins were transferred to polyvinylidene fluoride membranes $(0.45 \mu \mathrm{m})$. The membranes were blocked with 5\% non-fat milk (cat. no. LP0031; Solarbio Life Sciences, Inc.) in TBST (1\% Tween 20) at room temperature for $1 \mathrm{~h}$, and incubated with overnight with primary antibodies against anti-LIF (1:2,000 dilution; cat. no. NBP2-27406; Novus Biologicals, Ltd.), anti-p-STAT3 (Tyr705; 1:2,000 dilution; cat. no. CST9145; Cell Signaling Technology, Inc.), anti-STAT3 (1:2,000 dilution; cat. no. C-20; Santa Cruz Biotechnology, Inc.) and anti- $\beta$-actin (1:2,000 dilution; cat. no. A5441; Sigma-Aldrich; Merck KGaA) at $4^{\circ} \mathrm{C}$. Membranes were washed three times in TBST with $1 \%$ Tween-20 and then incubated with secondary goat anti-rabbit (1:2,000 dilution; cat. no. ab97051; Abcam, Inc.) or goat anti-rat (1:2,000 dilution; cat. no. ab97057; Abcam, Inc.) antibodies at room temperature for $1 \mathrm{~h}$. Blots were developed using enhanced chemiluminescence according to the manufacturer's instruction (cat. no. 1705060; Bio-Rad, Inc.). The intensity of each band was analyzed using Image J software (v.1.8.0.112; National Institutes of Health). $\beta$-actin was used as a loading control.

Reverse transcription-quantitative PCR (RT-qPCR). Total RNA from the tissues was purified using the RNeasy Kit (Qiagen, Inc.) according to the manufacturer's protocol, and it was reverse transcribed into cDNA using the reverse transcriptase and random hexamers found in the TaqMan $^{\mathrm{TM}}$ Reverse Transcription Reagents kit (Applied Biosystems; Thermo Fisher Scientific, Inc.) according to the manufacturer's protocol $\left(10 \mathrm{~min}\right.$ at $25^{\circ} \mathrm{C}, 120 \mathrm{~min}$ at $37^{\circ} \mathrm{C}, 5 \mathrm{~min}$ at $85^{\circ} \mathrm{C}$ and $4^{\circ} \mathrm{C}$ thereafter). Human LIF and $\beta$-actin cDNA levels were then quantified using the StepOnePlus ${ }^{\mathrm{TM}}$ Real-Time PCR System (Applied Biosystems; Thermo Fisher Scientific, Inc.). The $\mathrm{TaqMan}^{\mathrm{TM}}$ probes of LIF and $\beta$-actin were purchased from Applied Biosystems (Thermo Fisher Scientific, Inc; cat. no. for kit, 4351370). Each sample was measured in triplicate by RT-qPCR using the TaqMan ${ }^{\mathrm{TM}}$ PCR Master Mix (Applied Biosystems; Thermo Fisher Scientific, Inc.; cat. no. 4369016). The thermocycling conditions for qPCR were as follows: $95^{\circ} \mathrm{C}$ for $10 \mathrm{~min}$ (enzyme activation), followed by 40 cycles consisting of $95^{\circ} \mathrm{C}$ for $15 \mathrm{sec}$ (denaturing) and $60^{\circ} \mathrm{C}$ for 
1 min (annealing/extension). The expression level of the LIF gene was normalized to that of the internal reference gene $\beta$-actin (20). Relative gene expression was calculated using the $2^{-\triangle \Delta C q}$ method. Primer sequences were as follows: LIF forward, 5'-CCAACGTGACGGACTTCCC-3' and reverse, 5'-TACACG ACTATGCGGTACAGC-3'; and $\beta$-actin forward, 5'-CTAAGT CATAGTCCGCCTAGAAGCA-3' and reverse, 5'-TGGCAC CCAGCACAATGAA-3'.

Cell line and culture. The A549 cell line was purchased from the American Type Culture Collection. Cells were maintained in Dulbecco's modified Eagle's medium (DMEM; Gibco; Thermo Fisher Scientific, Inc.) with $10 \%$ fetal bovine serum (FBS; Gibco; Thermo Fisher Scientific, Inc.), penicillin $(100 \mathrm{IU} / \mathrm{ml})$ and streptomycin sulfate $(100 \mu \mathrm{g} / \mathrm{ml})$, and incubated at $37^{\circ} \mathrm{C}$ in a humidified incubator with $5 \% \mathrm{CO}_{2}$. Culture medium was replaced every 2-3 days and cells were harvested following treatment with $0.05 \%$ trypsin-EDTA solution (Gibco Thermo Fisher Scientific, Inc.) when $80-90 \%$ confluence was reached.

Cell proliferation and invasion assays. Cell proliferation was determined by Cell Counting Kit-8 (CCK-8; cat. no. HY-K0301; MedChemExpress, Inc.) assay following the manufacturer's protocol. A total of 1,000 cells in a volume of $100 \mu \mathrm{l}$ per well in a 96-well plate, in medium containing $10 \% \mathrm{FBS}$, were incubated for $24 \mathrm{~h}$ at $37^{\circ} \mathrm{C}$ and with $5 \% \mathrm{CO}_{2}$. Next, the different concentrations of $\operatorname{LIF}(100,200$ and $400 \mathrm{ng} / \mathrm{ml})$ (cat. no. 7734-LF-02; R\&D Systems, Inc.) were added on the following day and incubated for $48 \mathrm{~h}$. Untreated A549 cells were used as the control. At the end of incubation, the CCK- 8 reagent $(10 \mu \mathrm{l})$ was added to $90 \mu \mathrm{l}$ DMEM to generate a working solution, and $100 \mu \mathrm{l}$ per well was used for incubation for $2 \mathrm{~h}$, with absorbance measured at $450 \mathrm{~nm}$. Cell invasion assays were performed in 24-well Matrigel-coated Transwell invasion chambers with $8-\mu \mathrm{m}$ membrane pores (cat. no. 354480 ; Corning, Inc.). For the cell invasion assays, a cell suspension $\left(1 \times 10^{5}\right.$ cells $)$ in serum-free medium with different concentrations of $\operatorname{LIF}(100,200$ and $400 \mathrm{ng} / \mathrm{ml})$ was applied to the upper chamber. Untreated A549 cells were used as the control. The lower chamber contained DMEM supplemented with 30\% FBS. After $24 \mathrm{~h}$ of incubation, the invaded cells on the underside of the invasion chamber were stained with $0.5 \%$ crystal violet (cat. no. E607309; Shenggong, Inc.). The stained cells were counted under a microscope (TE2000; Nikon Corporation) and imaged from 10 different view fields for each membrane. This value represented the number of migrated cells through the membrane. Data were presented as the mean \pm SD of triplicate assays for each experimental condition.

Wound healing assay. A549 cells grown to $90 \%$ confluency in DMEM with $10 \%$ FBS were placed in medium containing $2 \% \mathrm{FBS}$ and incubated overnight at $37^{\circ} \mathrm{C}$. The cell layer was then scratched with a $200-\mu 1$ pipette tip and rinsed twice with PBS to remove floating cells and debris. The wound gap created by the scratch was measured with a microscope (TE2000; Nikon Corporation) immediately after the scratch and at $48 \mathrm{~h}$ after incubation with different concentrations of LIF (100, 200 and 400 ng/ml) (7734-LF-02; R\&D Systems, Inc.) in DMEM with 2\% FBS. Untreated A549 cells were used as the control. In the STAT3 blocking study, Stattic, a specific STAT3 inhibitor, was employed to block STAT3. A total of $200 \mathrm{ng} / \mathrm{ml} \mathrm{LIF}$ and $20 \mu \mathrm{M}$ Stattic (Sigma-Aldrich; Merck KGaA) were added to the A549 cells for incubation in DMEM with $2 \%$ FBS for $48 \mathrm{~h}$. All experiments were repeated three times.

Statistical analysis. Statistical analysis was performed using GraphPad Prism 7 (GraphPad Software). Data are expressed as the mean \pm standard deviation (SD). All expriments were independently repeated at last three times. An unpaired, two-tailed Student's t-test was used to statistically compare LIF and p-STAT3 expression between the adenocarcinoma and squamous carcinoma tissues. One-way ANOVA and Tukey's post hoc test were used for statistical comparisons in in vitro experiments, and repeated measures ANOVA with Bonferroni correction was used for the protein and mRNA expression comparisons among tumor, adjacent and normal samples. The correlation between the LIF and p-STAT3 IHC scores was evaluated by Pearson's correlation analysis. The association between LIF expression and the clinicopathological parameters of patients with NSCLC was analyzed using the $\chi^{2}$ test, except for lymph node metastasis, which was analyzed using Fisher's exact test. $\mathrm{P}<0.05$ was considered to indicate a statistically significant difference.

\section{Results}

LIF is overexpressed in NSCLC, specifically in the adenocarcinoma subtype. The mRNA level of LIF in NSCLC was measured. Using qPCR, the LIF mRNA levels in the tumor tissues were compared to those in the corresponding adjacent tissue and normal lung samples. As shown in Fig. 1A, the LIF mRNA level was significantly higher in the tumor tissues compared with that in the adjacent and normal tissues, and as expected, the LIF mRNA level in the adjacent tissue was higher compared with that in the normal tissue. LIF levels were also determined in NSCLS subtypes. The LIF mRNA level was significantly higher in adenocarcinoma tissues compared with that in squamous cell carcinoma tissues (Fig. 1B). Next, LIF protein expression in tumor, corresponding adjacent, and normal lung tissues were determined using western blot analysis, and corresponding protein bands derived from the same membrane. It was found that LIF expression was significantly higher in tumor tissues compared with that in the corresponding adjacent and normal tissues, which was consistent with the mRNA levels (Fig. 1C and D). Semi-quantitative analysis of LIF IHC staining revealed that LIF expression in NSCLC tumor tissues was significantly higher than that in the corresponding adjacent and normal tissues, and LIF staining score in adjacent tissues was also significantly higher than that in normal tissues (Fig. 1E and F). In addition, LIF protein expression was significantly higher in adenocarcinoma tissues compared with that in squamous cell carcinoma tissues (Fig. 1G). Thus, the present data indicate that LIF expression is elevated in NSCLC, particularly in the adenocarcinoma subtype.

LIF/STAT3 signaling is involved in the progression of NSCLC. Overexpression and activation of STAT3 are associated with the malignant behavior of carcinomas. To further explore 
A

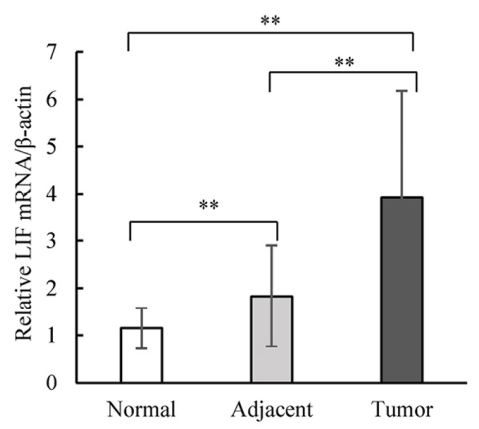

C
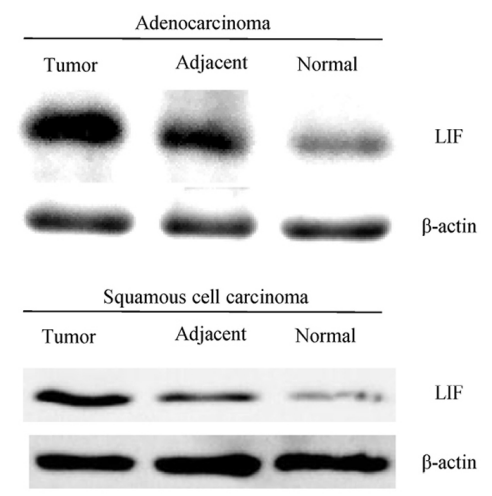

E
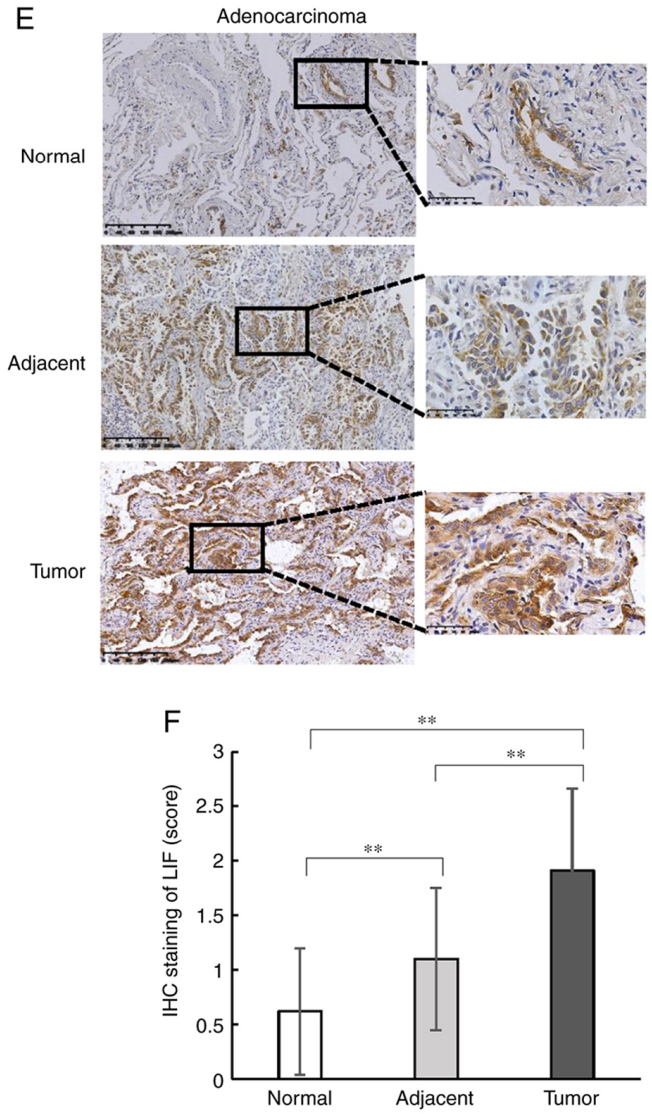

B

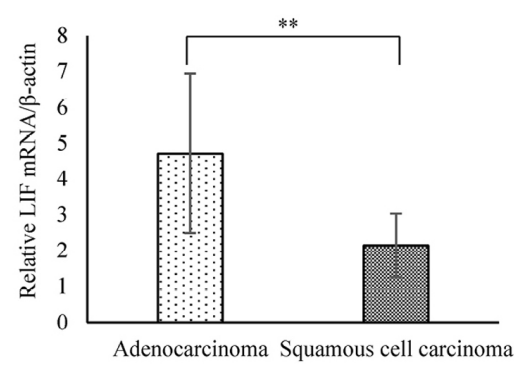

D
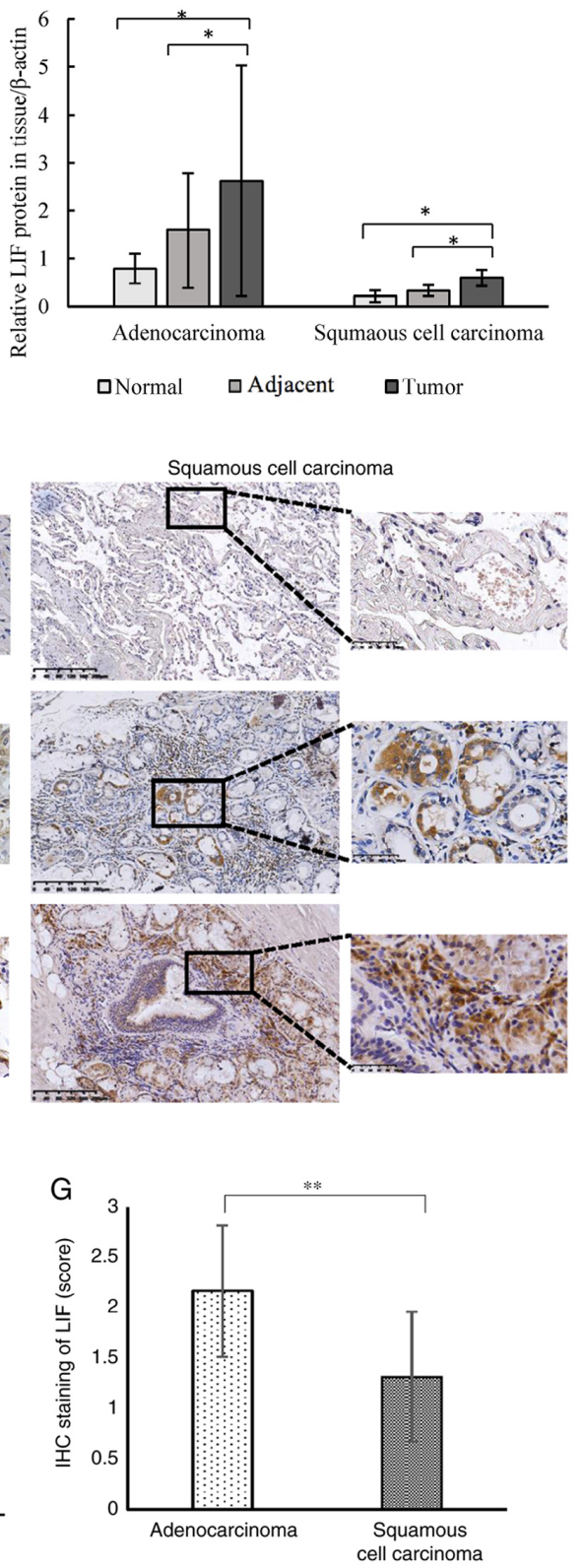

Figure 1. LIF expression in tissues derived from patients with NSCLC. (A) Relative mRNA expression of LIF/ $\beta$-actin in NSCLC tumor, adjacent and normal tissues. (B) Relative mRNA expression of LIF/ $\beta$-actin in adenocarcinoma and squamous cell carcinoma subtypes. (C) Western blot analysis of LIF expression in NSCLC tumor, adjacent and normal tissues. (D) Quantitative analysis of the western blot results for relative protein expression of LIF/ $\beta$-actin in tumor, adjacent and normal tissues in adenocarcinoma and squamous cell carcinoma subtypes. (E) IHC staining of LIF in tumor, adjacent and normal tissues of adenocarcinoma and squamous cell carcinoma subtypes (scale bar, 200 and $50 \mu \mathrm{m}$ ). (F) Quantitative analysis of IHC staining scores of LIF in tumor, adjacent and normal tissues. (G) Quantitative analysis of IHC staining scores of LIF in adenocarcinoma and squamous cell carcinoma subtypes. "P<0.05, ${ }^{* *} \mathrm{P}<0.01$. NSCLC, non-small cell lung cancer; LIF, leukemia inhibitory factor; IHC, immunohistochemistry. 
A

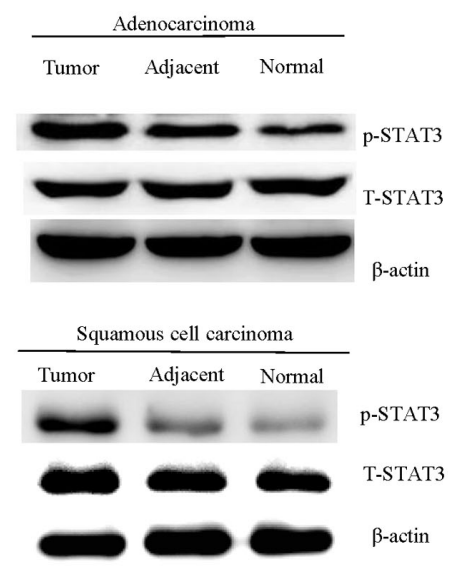

C

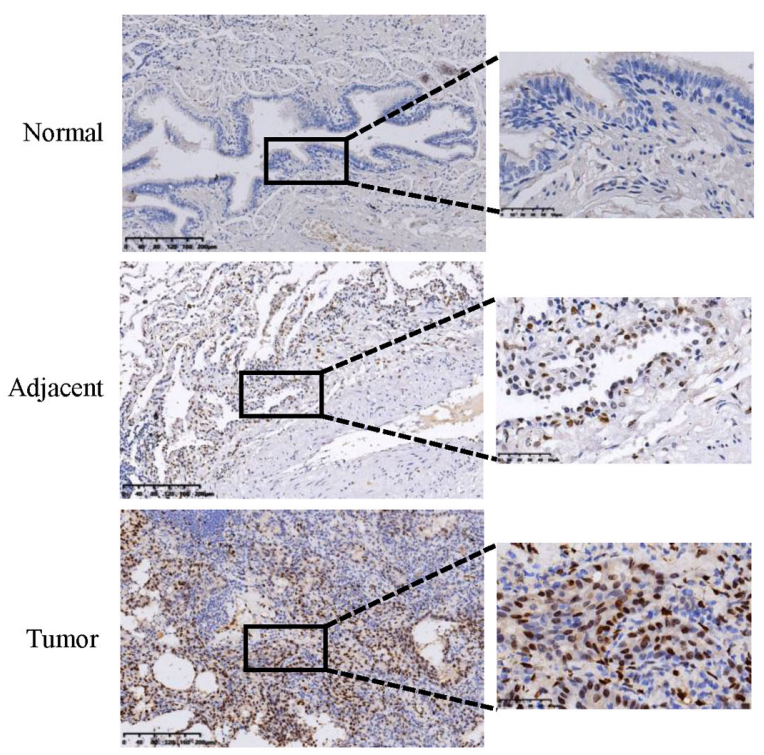

D

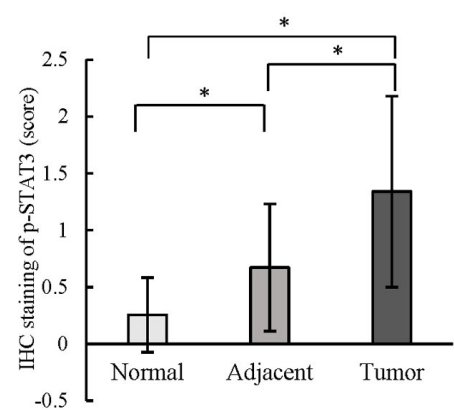

B

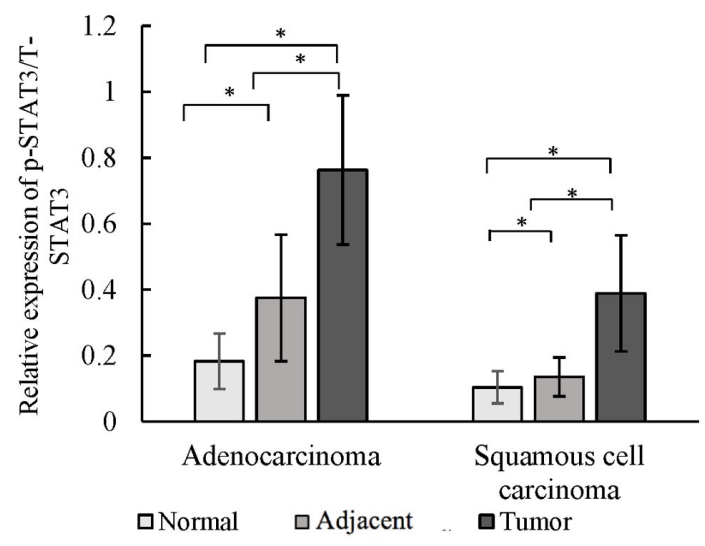

Squamous cell carcinoma

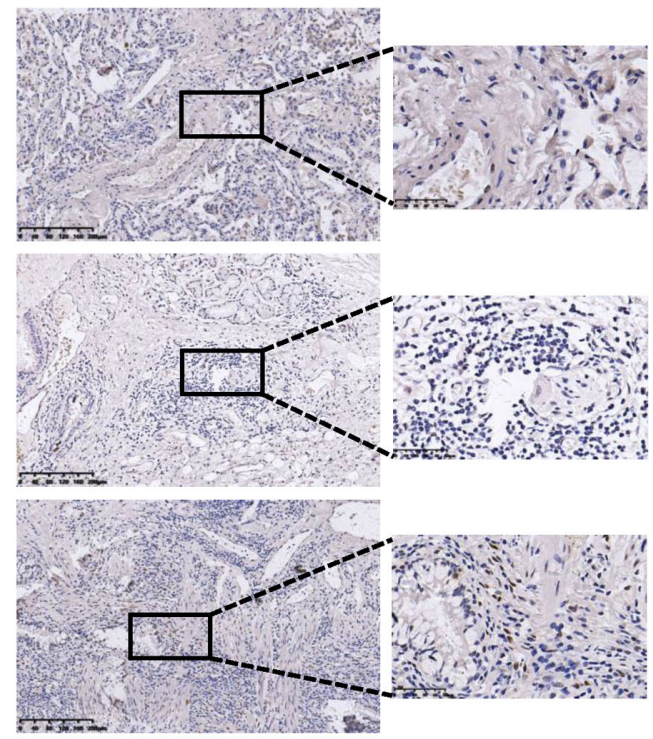

F

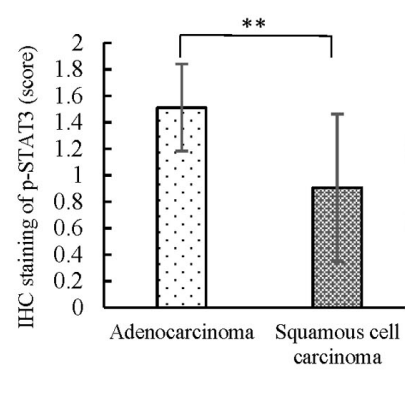

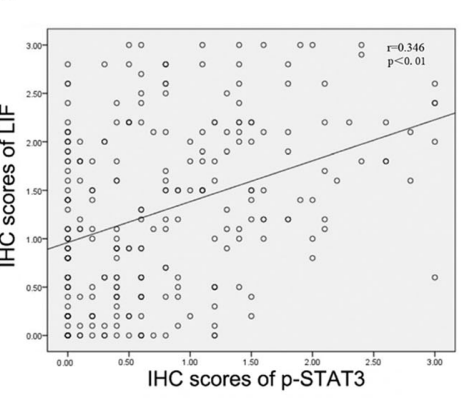

Figure 2. Expression of p-STAT3 expression in tissues derived from patients with NSCLC. (A) Western blot analysis of p-STAT3 and T-STAT3 expression in two subtypes of NSCLC. (B) Quantitative analysis of the p-STAT3/T-STAT3 expression ratio in tumor, adjacent and normal tissues of adenocarcinoma and squamous cell carcinoma subtypes. (C) IHC staining of p-STAT3 in tumor, adjacent and normal tissues of adenocarcinoma and squamous cell carcinoma subtypes (scale bar, 200 and $50 \mu \mathrm{m}$ ); (D) Quantitative analysis of IHC staining scores of p-STAT3 in tumor, adjacent and normal tissues. (E) Quantitative analysis of IHC staining scores of p-STAT3 in adenocarcinoma and squamous cell carcinoma subtypes. (F) Correlation analysis for IHC scores of p-STAT3 and LIF expression in tumor, adjacent and normal tissues. ${ }^{*} \mathrm{P}<0.05,{ }^{* *} \mathrm{P}<0.01$. STAT3, signal transducer and activator of transcription 3; p-, phosphorylated; T-, total; NSCLC, non-small cell lung cancer; IHC, immunohistochemistry.

the mechanism of LIF in NSCLC progression, western blot analysis was used to determine the activation of STAT3 in tissues with different LIF expression levels. It was found that the expression of p-STAT3 relative to that of total STAT3
(T-STAT3) in the tumor tissues from both adenocarcinoma and squamous cell carcinoma subtypes was significantly higher compared with that of the corresponding adjacent and normal tissues (Fig. 2A and B). IHC staining also showed that 
A

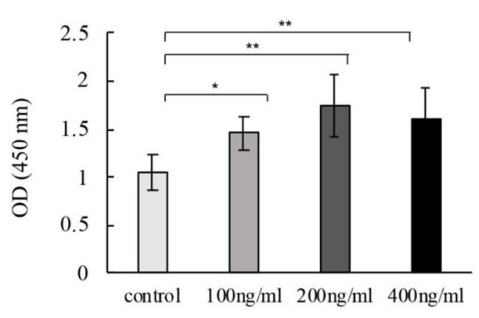

B

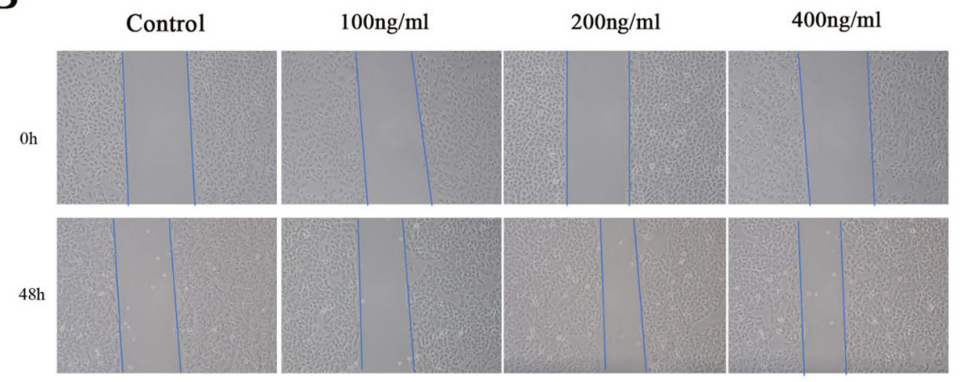

C

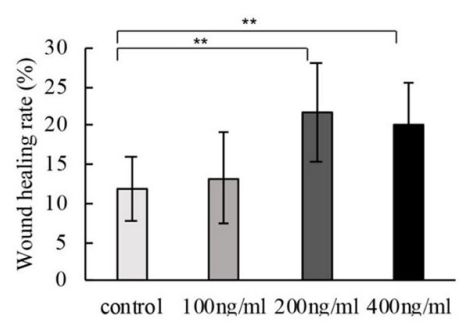

F

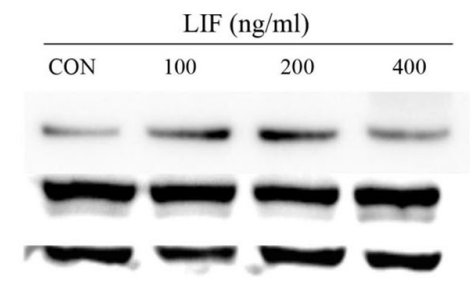

D

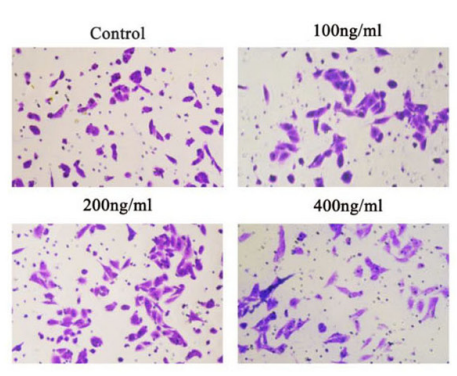

E

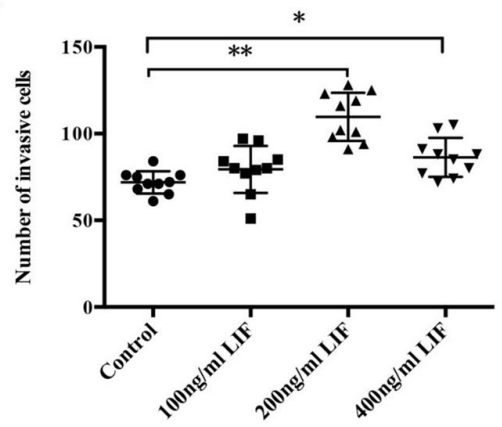

G

p-STAT3

T-STAT3

$\beta$-actin

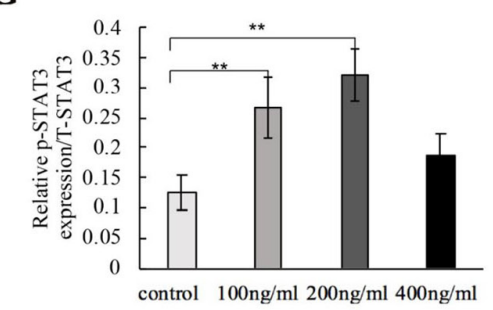

Figure 3. LIF promotes non-small cell lung cancer progression. (A) Cell proliferation was assessed by Cell Counting Kit-8 assay in A549 cells subjected to different levels of LIF stimulation. (B) Wound healing assays were used to evaluate the migration of A549 cells treated with different concentrations of LIF (magnification, x100). (C) Quantitative analysis of wound healing rate. (D) Transwell assays were used to measure the effects of LIF on the invasion of A549 cells (magnification, $x 400$ ). (E) Quantitative analysis of number of invasive cells. (F) Western blot analysis of p-STAT3 and T-STAT3 expression in A549 cells subjected to different levels of LIF stimulation. (G) Quantitative analysis of p-STAT3 and T-STAT3 expression. ${ }^{*} \mathrm{P}<0.05$, ${ }^{* *} \mathrm{P}<0.01$. STAT3, signal transducer and activator of transcription 3; p-, phosphorylated; T-, total; LIF, leukemia inhibitory factor; OD, optical density; CON, control.

p-STAT3 expression was higher in the tumor tissues compared with that in the matched adjacent and normal tissues (Fig. 2C). Semi-quantitative analysis revealed that p-STAT3 staining in tumor tissues of NSCLC was significantly higher compared with that in the corresponding normal tissues and adjacent tissues (Fig. 2D). p-STAT3 expression in adenocarcinoma tissues was higher than that in squamous cell carcinoma tissues (Fig. 2E). Correlation analysis showed that the IHC scores for p-STAT3 expression were positively correlated with LIF expression $(\mathrm{r}=0.346 ; \mathrm{P}<0.01)$ (Fig. $2 \mathrm{~F})$. These results indicate that STAT3 is activated in NSCLC, particularly in adenocarcinoma tissues, and its activation is associated with LIF expression.

LIF induces adenocarcinoma cell proliferation, invasion and migration, which is dependent on STAT3 activation. To further evaluate the effects of LIF on cell proliferation, invasion and migration, NSCLC-derived A549 adenocarcinoma cells were treated with LIF. It was found that LIF treatment significantly stimulated A549 cell proliferation (Fig. 3A). Wound healing assay revealed that high concentrations of LIF (200 and $400 \mathrm{ng} / \mathrm{ml}$ ) significantly increased the invasion ability of A549 cells compared with that of the untreated control and low LIF concentration $(100 \mathrm{ng} / \mathrm{ml})$ groups. No significant difference was found for $100 \mathrm{ng} / \mathrm{ml}$ (Fig. 3B and C). Transwell assays showed that high concentrations of LIF promoted invasion of A549 cells (Fig. 3D). Quantitative analysis revealed that the number of invasive cells significantly increased with LIF treatment (200 and $400 \mathrm{ng} / \mathrm{ml}$ ) compared with that of the untreated control (Fig. 3E).

To explore the association of STAT3 activation and LIF overexpression in tissues derived from patients with NSCLC, the effects of LIF treatment on p-STAT3 expression relative to T-STAT3 expression were measured in A549 cells. Western blot analysis showed that LIF treatment (100 and $200 \mathrm{ng} / \mathrm{ml}$ ) significantly increased the p-STAT3/T-STAT3 ratio compared with that of the untreated control. Higher concentrations $(400 \mathrm{ng} / \mathrm{ml})$ had a tendency to activate $\mathrm{p}$-STAT3 expression $(\mathrm{P}>0.05)$, but the activity was the highest at $200 \mathrm{ng} / \mathrm{ml}$ (Fig. 3F and G). Stattic, a specific STAT3 inhibitor, prevents 
A
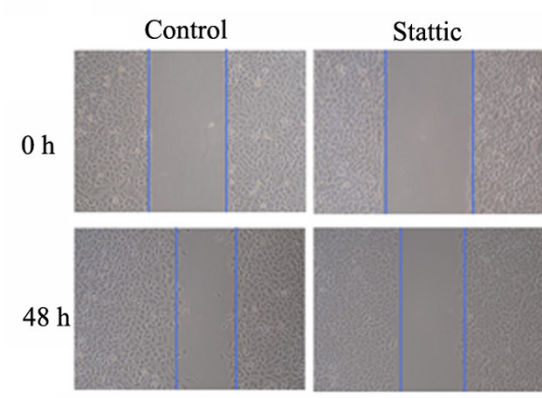

\section{Control}

C

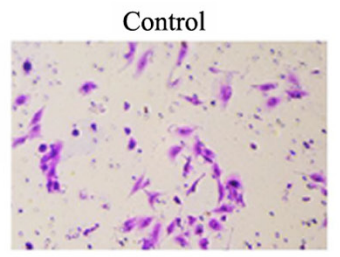

\section{LIF}

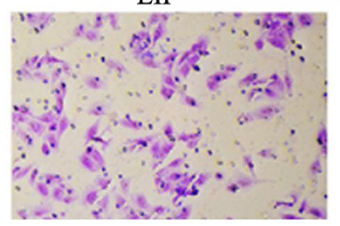

LIF
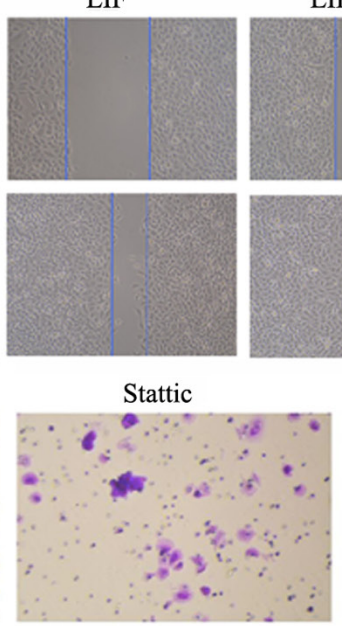

LIF+Stattic

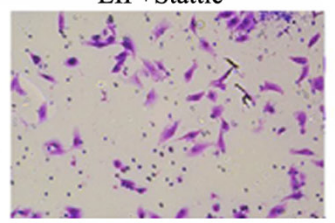

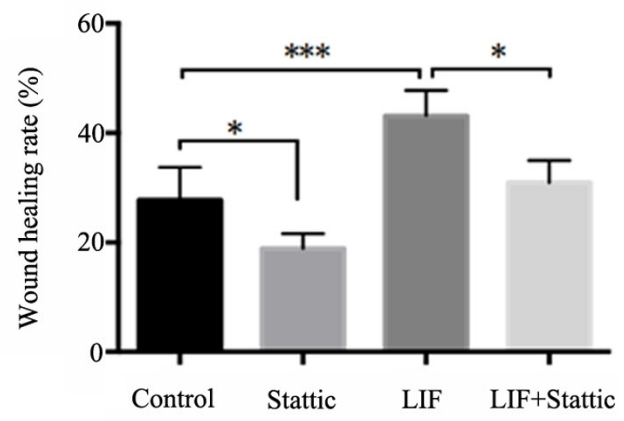

$\mathrm{D}$

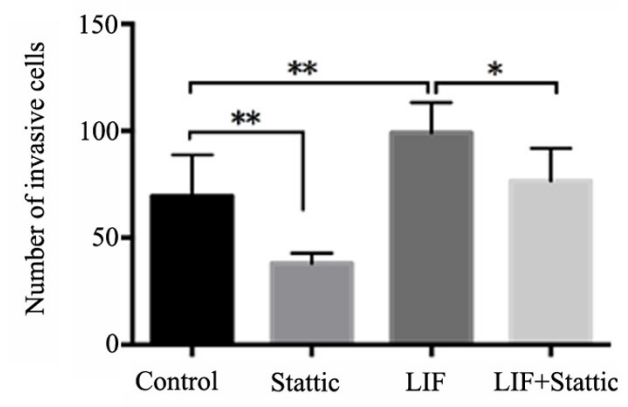

Figure 4. Inhibition of signal transducer and activator of transcription 3 with Stattic abolishes the effects of LIF on A549 cell migration and invasion. (A) Wound healing assays were used to evaluate the migration of A549 cells following LIF stimulation $(200 \mathrm{ng} / \mathrm{ml})$ with or without Stattic (20 $\mu \mathrm{M})(\mathrm{magnifica}-$ tion, x100). (B) Quantitative analysis of wound healing rate. (C) Transwell assays were used to measure the effects of LIF stimulation (200 ng/ml) with or without Stattic $(20 \mu \mathrm{M})$ on the invasion of A549 cells (magnification, $\mathrm{x} 400)$. (D) Quantitative analysis of number of invasive cells. ${ }^{*} \mathrm{P}<0.05,{ }^{* *} \mathrm{P}<0.01{ }^{, * * *} \mathrm{P}<0.001$. LIF, leukemia inhibitory factor.
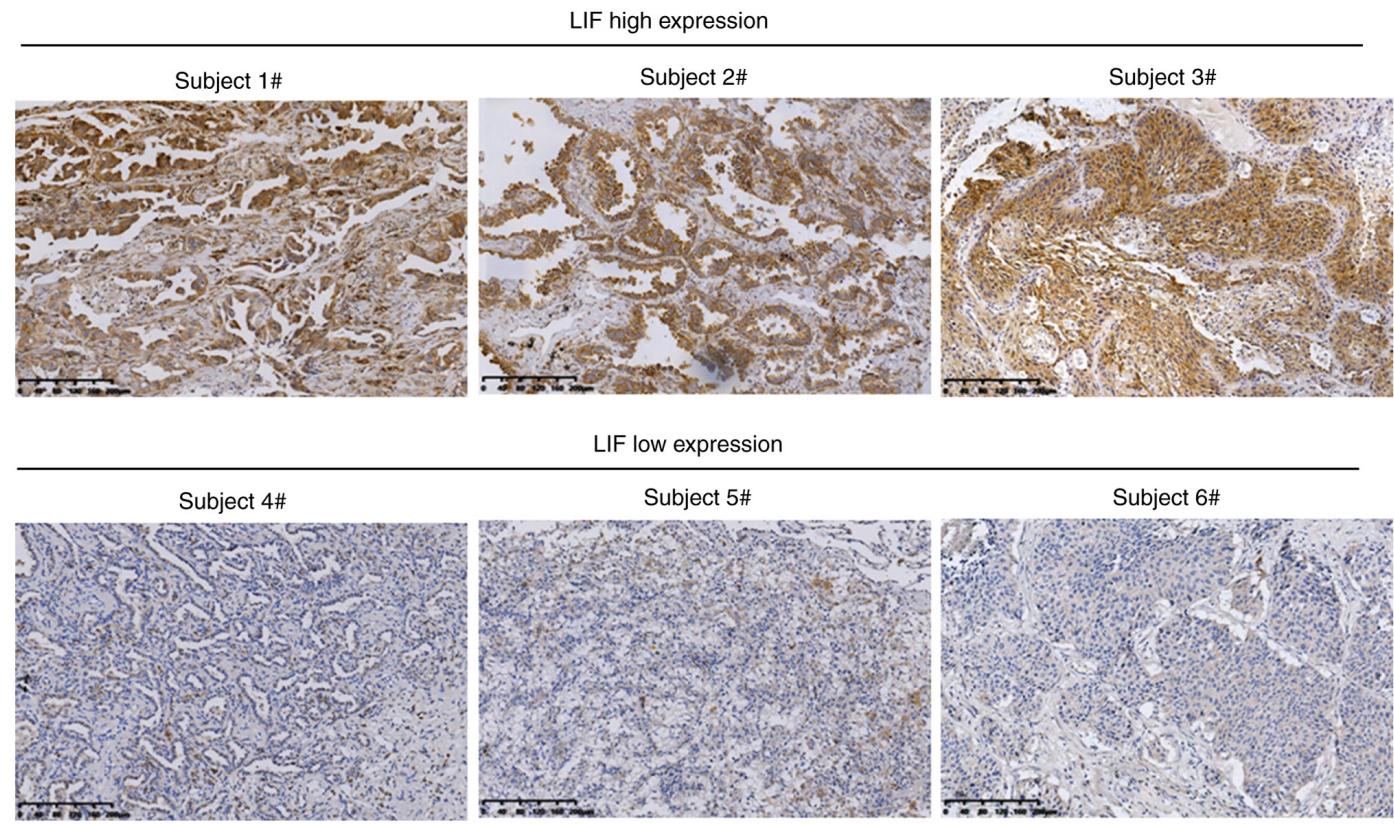

Figure 5. Representative image of LIF expression in patients with non-small cell lung cancer. Scale bar, $200 \mu \mathrm{m}$. LIF, leukemia inhibitory factor.

activation, dimerization and nuclear translocation of STAT3 by interacting with the $\mathrm{SH} 2$ domain (21). It was also used to determine the effects of blocking STAT3 following LIF treatment (200 $\mathrm{ng} / \mathrm{ml}$ ) on cell invasion and migration. Wound healing assays showed that Stattic decreased A549 cell migration both in the control group treated only with Stattic and in the group treated with LIF and Stattic (Fig. 4A and B). Transwell assays showed that Stattic significantly attenuated A549 cell invasion. There was no significant difference between the control and LIF + Stattic groups (Fig. 4C and D). Thus, LIF plays a role in adenocarcinoma cell proliferation, invasion and migration through activating the LIF/STAT3 signaling pathway. 
Table I. Association between LIF expression and clinicopathological characteristics in 105 non-small cell lung cancer tissues.

\begin{tabular}{|c|c|c|c|c|}
\hline \multirow[b]{2}{*}{ Characteristics } & \multirow[b]{2}{*}{ Patients, $\mathrm{n}$} & \multicolumn{2}{|c|}{ LIF expression level (score) } & \multirow[b]{2}{*}{ P-value } \\
\hline & & $\operatorname{Low}^{a}(n=36)$ & $\operatorname{High}^{\mathrm{b}}(\mathrm{n}=69)$ & \\
\hline \multicolumn{5}{|l|}{ Age, years } \\
\hline$<60$ & 40 & 9 & 31 & \multirow[t]{2}{*}{0.058} \\
\hline$\geq 60$ & 65 & 27 & 38 & \\
\hline \multicolumn{5}{|l|}{ Sex } \\
\hline Male & 45 & 18 & 27 & \multirow[t]{2}{*}{0.285} \\
\hline Female & 60 & 18 & 42 & \\
\hline \multicolumn{5}{|l|}{ Smoking status } \\
\hline Smoker & 60 & 20 & 40 & \multirow[t]{2}{*}{0.812} \\
\hline Non-smoker & 45 & 16 & 29 & \\
\hline \multicolumn{5}{|l|}{ Histological type } \\
\hline Squamous cell carcinoma & 36 & 28 & 8 & \multirow[t]{2}{*}{$<0.0001^{\mathrm{c}}$} \\
\hline Adenocarcinoma & 69 & 8 & 61 & \\
\hline \multicolumn{5}{|l|}{ Tumor stage } \\
\hline I-II & 86 & 33 & 53 & \multirow[t]{2}{*}{$0.047^{\mathrm{c}}$} \\
\hline III-IV & 19 & 3 & 16 & \\
\hline \multicolumn{5}{|l|}{ Lymph node metastasis } \\
\hline Yes & 10 & 0 & 10 & \multirow[t]{2}{*}{$0.012^{\mathrm{c}, \mathrm{d}}$} \\
\hline No & 95 & 36 & 59 & \\
\hline \multicolumn{5}{|l|}{ Tumor size, $\mathrm{cm}$} \\
\hline$<2$ & 83 & 26 & 57 & \multirow[t]{2}{*}{0.214} \\
\hline$\geq 2$ & 22 & 10 & 12 & \\
\hline \multicolumn{5}{|l|}{ Differentiation } \\
\hline Well/moderate & 88 & 30 & 58 & \multirow[t]{2}{*}{0.924} \\
\hline Poor & 17 & 6 & 11 & \\
\hline
\end{tabular}

${ }^{\mathrm{a}}$ Low: Score, 0-1.49; ${ }^{\mathrm{b}} \mathrm{High}$ : Score, 1.50-3.00; ${ }^{\mathrm{c}} \mathrm{P}<0.05$; ${ }^{\mathrm{d}}$ Fisher's exact test. All other data were analyzed by $\chi^{2}$ test. LIF, leukemia inhibitory factor.

Association between LIF expression and clinical features of patients with NSCLC. The present study explored the association between LIF expression in tumor tissues and the clinical features of patients with NSCLC. Potentially due to the stage and histological type, LIF expression varied greatly among NSCLC tissues (Fig. 5). Based on the IHC score for LIF expression, the cohort of 105 patients with NSCLC was divided into LIF ${ }^{\text {high }}$ and LIF ${ }^{\text {low }}$ groups (low, 0-1.49; high, 1.50-3.00). As shown in Table I, high LIF expression significantly correlated with histological type $(\mathrm{P}<0.0001)$ and aggressive tumor characteristics, including lymph node metastasis $(\mathrm{P}=0.012)$ and advanced tumor stage $(\mathrm{P}=0.043)$. These data suggest that LIF expression is negatively correlated with prognosis in patients with NSCLC.

\section{Discussion}

LIF was originally shown to regulate the differentiation of myeloid leukemia cells (22), and was thus named 'leukemia inhibitory factor'. However, other studies have since demonstrated that LIF can also facilitate the development and progression of a variety of solid tumors $(11,12,23)$. In fact, LIF overexpression has been found in breast, colorectal, head and neck, and ovarian cancer, as well as in melanoma, nasopharyngeal carcinoma and pancreatic adenocarcinoma. High expression of LIF in tumors is linked to poor clinical outcomes in patients with cancer (10-12,23-26). Increased LIF expression also contributes to tumor resistance to chemotherapy and radiotherapy $(11,12)$. These lines of evidence strongly suggest that LIF can promote tumorigenesis, particularly in solid tumors. However, the role of LIF in lung cancer remained unknown.

The present study provides evidence that overexpression of LIF in NSCLC tissue is associated with cell metastasis to the lymph node; patients who underwent surgery had earlier stages and fewer lymph node metastases, and a statistically significant correlation was noted between LIF expression and lymph node metastasis in enrolled patients. These results indicate that LIF plays an important role in the tumorigenesis of NSCLC, similar to its previously reported role in other solid tumors, as aforementioned. Since LIF overexpression can increase the proliferation rate of cultured cancer cells, the growth rate of 
xenograft tumors and the metastasis of multiple human tumor types $(10-12,23,24)$. The present study also evaluated the effects of LIF on a human lung cancer cell line in vitro. It was found that LIF stimulation promoted cancer cell proliferation, migration and invasion, further suggesting that LIF induces metastasis of lung cancer. These in vitro results are consistent with findings from clinical specimens, which showed that LIF is overexpressed in tumor tissues along with activation of the STAT3 pathway. Together, these findings demonstrate that LIF is important for lung cancer progression. The present findings also provide a useful guide in clinical practice, since high LIF expression could be used a marker of regional lymph node metastasis in patients with NSCLC.

The molecular mechanisms of LIF's role in promoting tumor metastasis are not fully understood. LIF functions in an autocrine and/or paracrine manner through binding to the LIF receptor (LIF-R) complex (which is composed of LIF-R and gp-130), which in turn activates certain signaling pathways, including the PI3K/AKT and JAK/STAT3 pathways (27-29). STAT3 is a critical downstream effector of LIF signaling in numerous types of cells and tissues, such as embryonic stem cells and monocytes (27,30). LIF expression in colorectal cancer cells can be induced by hypoxia and transcription factor hypoxia-inducible factor- $2 \alpha$ (18). In addition, LIF can negatively regulate p53 in colorectal cancer cells, which is associated with its effect of increasing resistance of cancer cells to chemotherapy and radiotherapy (31). Yang et al (32) showed that STAT5B and STAT6 could be effective prognostic biomarkers of survival in patients with NSCLC, and that STAT2 may be a promising therapeutic target for the treatment of NSCLC, and in particular lung adenocarcinoma. STAT3 is frequently activated in multiple types of human cancer, and is crucial to the survival and growth of tumor cells (33-36). Jiang et al (37) reported that immunoreactivity of p-STAT3 was significantly increased in lung cancer tissue compared with that of normal tissue. The present study showed a high level of p-STAT3 expression in tumor tissues compared with that of the corresponding adjacent and normal lung tissues. Furthermore, the expression of p-STAT3 was positively correlated with LIF expression in NSCLC tissue samples. LIF (200 and $400 \mathrm{ng} / \mathrm{ml}$ ) stimulation also induced STAT3 phosphorylation in A549 cells, which is correlated with LIF expression levels in NSCLC tissues. The STAT3-specific inhibitor Stattic inhibited cell migration and invasion. These findings suggest that LIF promotes the development and progression of NSCLC through activating the LIF/STAT3 signaling pathway, although other pathways could also be involved.

Although there are significant differences between lung adenocarcinoma and lung squamous cell carcinoma in regards to clinical features, gene mutations, immune checkpoints, cytokines and non-coding RNA expression (38-41), the comparison of LIF expression between these two subtypes of NSCLC has not been reported to date. The present results show that LIF and p-STAT3 expression levels are higher in lung adenocarcinoma compared with those of lung squamous cell carcinoma. However, the detailed molecular mechanisms underlying these differences need to be further studied.

In conclusion, the present results demonstrate that LIF overexpression can promote NSCLC development through activating the LIF/STAT3 signaling pathway. Therefore, LIF may serve as a potential prognostic marker for patients with NSCLC, and LIF signaling pathways could be potential targets for anticancer drug discovery for NSCLC.

\section{Acknowledgements}

Not applicable.

\section{Funding}

The present study was funded by the Nature Scientific Foundation of Ningbo City (grant no. 2019A610229), and the Major Project of Diseases Prevention and Treatment of Traditional Chinese Medicine of Zhejiang Province (grant no. 2018ZY010). The funding bodies had no role in the design of the study; in the collection, analysis or interpretation of the data; or in the writing of the manuscript.

\section{Availability of data and materials}

The datasets used and/or analyzed during the current study are available from the corresponding author on reasonable request.

\section{Authors' contributions}

HW performed the IHC staining, participated in patient enrollment and follow-up, performed the statistical analysis, and drafted the manuscript. SS performed the RT-qPCR, western blotting and IHC staining. $\mathrm{MJ}$ and $\mathrm{KH}$ participated in patient enrollment, clinical data analysis and IHC staining. LC performed the cell culture and in vitro experiments, including cell proliferation and invasion assays, and the wound-healing assay. WY conceived the study, participated in its design and coordination, and helped to draft the manuscript. All authors read and approved the final manuscript. HW, SS, LC and WY confirm the authenticity of all the raw data.

\section{Ethics approval and consent to participate}

The present study was approved by the Ethics Committee of Yinzhou People's Hospital (approval no. 2018003). Written informed consent to participate was obtained from all patients.

\section{Patient consent for publication}

Not applicable.

\section{Competing interests}

The authors declare that they have no competing interests.

\section{References}

1. Bray F, Ferlay J, Soerjomataram I, Siegel RL, Torre LA and Jemal A: Global cancer statistics 2018: GLOBOCAN estimates of incidence and mortality worldwide for 36 cancers in 185 countries. CA Cancer J Clin 68: 394-424, 2018.

2. Xiong D, Zhu SQ, Wu YB, Jin C, Jiang JH, Liao YF, Long X, $\mathrm{Wu} \mathrm{HB}, \mathrm{Xu}$ JJ, Li JJ and Ding JY: Ring finger protein 38 promote non-small cell lung cancer progression by endowing cell EMT phenotype. J Cancer 9: 841-850, 2018. 
3. Pirker R: Conquering lung cancer: Current status and prospects for the future. Pulmonology 26: 283-290, 2020.

4. Scrima M,Zito Marino F, Oliveira DM, Marinaro C, La Mantia E, Rocco G, De Marco C, Malanga D, De Rosa N, Rizzuto A, et al: Aberrant signaling through the HER2-ERK1/2 pathway is predictive of reduced disease-free and overall survival in early stage non-small cell lung cancer (NSCLC) Patients. J Cancer 8: 227-239, 2017

5. Rotow $\mathrm{J}$ and Bivona TG: Understanding and targeting resistance mechanisms in NSCLC. Nat Rev Cancer 17: 637-658, 2017.

6. Nicola NA and Babon JJ: Leukemia inhibitory factor (LIF). Cytokine Growth Factor Rev 26: 533-544, 2015.

7. Dahéron L, Opitz SL, Zaehres H, Lensch MW, Andrews PW, Itskovitz-Eldor J and Daley GQ: LIF/STAT3 signaling fails to maintain self-renewal of human embryonic stem cells. Stem Cells 22: 770-778, 2004.

8. Niwa H, Ogawa K, Shimosato D and Adachi K: A parallel circuit of LIF signalling pathways maintains pluripotency of mouse ES cells. Nature 460: 118-122, 2009.

9. Pera MF and Tam PP: Extrinsic regulation of pluripotent stem cells. Nature 465: 713-720, 2010.

10. Wysoczynski M, Miekus K, Jankowski K, Wanzeck J, Bertolone S, Janowska-Wieczorek A, Ratajczak J and Ratajczak MZ: Leukemia inhibitory factor: A newly identified metastatic factor in rhabdomyosarcomas. Cancer Res 67: 2131-2140, 2007.

11. Liu SC, Tsang NM, Chiang WC, Chang KP, Hsueh C, Liang Y, Juang JL, Chow KP and Chang YS: Leukemia inhibitory factor promotes nasopharyngeal carcinoma progression and radioresistance. J Clin Invest 123: 5269-5283, 2013.

12. Yu H, Yue X, Zhao Y, Li X, Wu L, Zhang C, Liu Z, Lin K, $\mathrm{Xu}$-Monette $\mathrm{ZY}$, Young KH, et al: LIF negatively regulates tumour-suppressor p53 through Stat3/ID1/MDM2 in colorecta cancers. Nat Commun 5: 5218, 2014.

13. Lin TA, Wu TS, Li YJ, Yang CN, Illescas Ralda MM and Chang HH: Role and mechanism of LIF in oral squamous cell carcinoma progression. J Clin Med 9: 295, 2020.

14. Hergovich A: YAP-Hippo signalling downstream of leukemia inhibitory factor receptor: Implications for breast cancer. Breast Cancer Res 14: 326, 2012

15. Chen D, Sun Y, Wei Y, Zhang P, Rezaeian AH, Teruya-Feldstein J, Gupta S, Liang H, Lin HK, Hung MC and Ma L: LIFR is a breast cancer metastasis suppressor upstream of the Hippo-YAP pathway and a prognostic marker. Nat Med 18: 1511-1517, 2012.

16. Humbert L, Ghozlan M, Canaff L, Tian J and Lebrun JJ: The leukemia inhibitory factor (LIF) and p21 mediate the TGF $\beta$ tumor suppressive effects in human cutaneous melanoma. BMC Cancer 15: 200, 2015

17. Luo Q, Wang C, Jin G, Gu D, Wang N, Song J, Jin H, Hu F, Zhang Y, Ge T, et al: LIFR functions as a metastasis suppressor in hepatocellular carcinoma by negatively regulating phosphoinositide 3-kinase/AKT pathway. Carcinogenesis 36: 1201-1212, 2015

18. Wu L, Yu H, Zhao Y, Zhang C, Wang J, Yue X, Yang Q and $\mathrm{Hu} \mathrm{W}$ : HIF-2 $\alpha$ mediates hypoxia-induced LIF expression in human colorectal cancer cells. Oncotarget 6: 4406-4417, 2015.

19. Shiao YH, Palli D, Caporaso NE, Alvord WG, Amorosi A, Nesi G, Saieva C, Masala G, Fraumeni JF Jr and Rice JM: Genetic and immunohistochemical analyses of p53 independently predict regional metastasis of gastric cancers. Cancer Epidemiol Biomarkers Prev 9: 631-633, 2000.

20. Morse DL, Carroll D, Weberg L, Borgstrom MC, Ranger-Moore J and Gillies RJ: Determining suitable internal standards for mRNA quantification of increasing cancer progression in human breast cells by real-time reverse transcriptase polymerase chain reaction. Anal Biochem 342: 69-77, 2005.

21. Guha P, Gardell J, Darpolor J, Cunetta M, Lima M, Miller G Espat NJ, Junghans RP and Katz SC: STAT3 inhibition induces Bax-dependent apoptosis in liver tumor myeloid-derived suppressor cells. Oncogene 38: 533-548, 2019.

22. Moreau JF, Donaldson DD, Bennett F, Witek-Giannotti J, Clark SC and Wong GG: Leukaemia inhibitory factor is identical to the myeloid growth factor human interleukin for DA cells. Nature 336: 690-692, 1988

23. Li X, Yang Q, Yu H, Wu L, Zhao Y, Zhang C, Yue X, Liu Z, Wu H, Haffty BG, et al: LIF promotes tumorigenesis and metastasis of breast cancer through the AKT-mTOR pathway. Oncotarget 5 : $788-801,2014$
24. Kuphal S, Wallner S and Bosserhoff AK: Impact of LIF (leukemia inhibitory factor) expression in malignant melanoma. Exp Mol Pathol 95: 156-165, 2013

25. Wang D, Liu K, Yang Y, Wang T, Rao Q, Guo W and Zhang Z: Prognostic value of leukemia inhibitory factor and its receptor in pancreatic adenocarcinoma. Future Oncol 16: 4461-4473, 2020.

26. McLean K, Tan L, Bolland DE, Coffman LG, Peterson LF, Talpaz M, Neamati N and Buckanovich RJ: Leukemia inhibitory factor functions in parallel with interleukin- 6 to promote ovarian cancer growth. Oncogene 38: 1576-1584, 2019.

27. Takahashi Y, Takahashi M, Carpino N, Jou ST, Chao JR, Tanaka S, Shigeyoshi Y, Parganas E and Ihle JN: Leukemia inhibitory factor regulates trophoblast giant cell differentiation via Janus kinase 1-signal transducer and activator of transcription 3-suppressor of cytokine signaling 3 pathway. Mol Endocrinol 22: 1673-1681, 2008.

28. Heinrich PC, Behrmann I, Haan S, Hermanns HM, Müller-Newen G and Schaper F: Principles of interleukin (IL)-6-type cytokine signalling and its regulation. Biochem J 374: 1-20, 2003.

29. Watanabe S, Umehara H, Murayama K, Okabe M, Kimura T and Nakano T: Activation of Akt signaling is sufficient to maintain pluripotency in mouse and primate embryonic stem cells. Oncogene 25: 2697-2707, 2006.

30. Metcalf D: The unsolved enigmas of leukemia inhibitory factor. Stem Cells 21: 5-14, 2003

31. Yue X, Zhao Y, Zhang C, Li J, Liu Z, Liu J and Hu W: Leukemia inhibitory factor promotes EMT through STAT3-dependent miR-21 induction. Oncotarget 7: 3777-3790, 2016.

32. Yang M, Chen H, Zhou L, Chen K and Su F: Expression profile and prognostic values of STAT family members in non-small cell lung cancer. Am J Transl Res 11: 4866-4880, 2019.

33. Lassmann S, Schuster I, Walch A, Göbel H, Jütting U, Makowiec F, Hopt U and Werner M: STAT3 mRNA and protein expression in colorectal cancer: Effects on STAT3-inducible targets linked to cell survival and proliferation. J Clin Pathol 60: 173-179, 2007.

34. Lin L, Liu A, Peng Z, Lin HJ, Li PK, Li C and Lin J: STAT3 is necessary for proliferation and survival in colon cancer-initiating cells. Cancer Res 71: 7226-7237, 2011.

35. Grivennikov S, Karin E, Terzic J, Mucida D, Yu GY, Vallabhapurapu S, Scheller J, Rose-John S, Cheroutre H, Eckmann L and Karin M: IL-6 and Stat 3 are required for survival of intestinal epithelial cells and development of colitis-associated cancer. Cancer Cell 15: 103-113, 2009.

36. Bollrath J, Phesse TJ, von Burstin VA, Putoczki T, Bennecke M, Bateman T, Nebelsiek T, Lundgren-May T, Canli O, Schwitalla S, et al: gp130-mediated Stat3 activation in enterocytes regulates cell survival and cell-cycle progression during colitis-associated tumorigenesis. Cancer Cell 15: 91-102, 2009.

37. Jiang R, Jin Z, Liu Z, Sun L, Wang L and Li K: Correlation of activated STAT3 expression with clinicopathologic features in lung adenocarcinoma and squamous cell carcinoma. Mol Diagn Ther 15: 347-352, 2011.

38. Tian Y, Yu M, Sun L, Liu L, Wang J, Hui K, Nan Q, Nie X, Ren Y and Ren X: Distinct Patterns of mRNA and lncRNA expression differences between lung squamous cell carcinoma and adenocarcinoma. J Comput Biol 27: 1067-1078, 2020.

39. Zhang XC, Wang J, Shao GG, Wang Q, Qu X, Wang B, Moy C, Fan Y, Albertyn Z, Huang X, et al: Comprehensive genomic and immunological characterization of Chinese non-small cell lung cancer patients. Nat Commun 10: 1772, 2019.

40. Kim H, Kwon HJ, Park SY, Park Y, Park E and Chung JH: Clinicopathological analysis and prognostic significance of programmed cell death-ligand 1 protein and mRNA expression in non-small cell lung cancer. PLoS One 13: e0198634, 2018.

41. Liu S, Wang X, Qin W, Genchev GZ and Lu H: Transcription factors contribute to differential expression in cellular pathways in lung adenocarcinoma and lung squamous cell carcinoma. Interdiscip Sci 10: 836-847, 2018.

This work is licensed under a Creative Commons Attribution-NonCommercial-NoDerivatives 4.0 International (CC BY-NC-ND 4.0) License. 